\title{
Gobierno abierto y competencias digitales: Transformando la administración pública para afrontar los retos del nuevo paradigma
}

\author{
Miguel Salvador Serna* \\ Olga Ramírez Hernández**
}

\section{Recibido: 11/01//2016 / Aceptado: 23/03/2016}

Resumen. El artículo plantea una contribución al debate académico sobre el surgimiento del Gobierno Abierto y los requerimientos de transformación de las Administraciones Públicas para afrontar a los retos que plantea. El texto, en base a la aportación de reflexiones y evidencias, se centra en el ámbito de los profesionales del sector público y la generación de competencias digitales. El marco analítico se combina con la presentación de evidencias basadas en dos estudios de caso de innovación en gestión de recursos humanos. Los resultados obtenidos permiten ilustrar las sinergias que se dan entre el Gobierno Abierto y el desarrollo de las competencias digitales, y cómo con ello se puede orientar la mejora las capacidades institucionales de las Administraciones públicas

Palabras clave: Gobierno abierto, competencias digitales, gestión de recursos humanos e innovación.

\section{Open Government and digital skills: transforming public administration to face new paradigm's challenges}

\begin{abstract}
The article aims to contribute to the academic debate on the emergence of Open Government with evidences and reflections on the required transformations to adapt Public Administrations to the new paradigm's challenges. The text focuses on the field of public sector's professionals and the generation of e-skills. The analytical framework is combined with the introduction of evidences based on two case studies on innovative initiatives in human resource management. The results obtained illustrate the synergies that exist between the Open Government and the development of digital skills, and how with both can orient institutional capacity improvement of Public Administrations.
\end{abstract}

Keywords: Open government, digital skills, human resource management and innovation.

Sumario. 1. Introducción. 2. Gobierno abierto y cambios internos en la administración. 3. Competencias digitales y gobierno abierto. 4. Dos experiencias considerar. 5. Análisis de las experiencias. 6. Conclusiones. Bibliografía.

Cómo citar: Salvador Serna, M., Ramírez Hernández, O., «Gobierno abierto y competencias digitales: Transformando la administración pública para afrontar los retos del nuevo paradigma», en Cuadernos de Gobierno y Administración Pública 3-1, 00-00.

\footnotetext{
* Universitat Pompeu Fabra miquel.salvador@upf.edu

** Generalitat de Cataluya

olgarahe@gmail.com
} 


\section{Introducción}

La incorporación intensiva de las tecnologías de la información y la comunicación (TIC) en las organizaciones públicas y su reflejo en los referentes del Gobierno Electrónico y el Gobierno Digital, asociados a la administración electrónica, han sido algunos de los términos de referencia para ilustrar unos procesos de cambio que pretendían tanto mejorar la atención a la ciudadanía como introducir mejoras internas principalmente en clave de eficacia y eficiencia. Sin embargo en muchas ocasiones las expectativas generadas distan de las realidades observadas.

Más allá de su desigual desarrollo y resultados, buena parte del debate tiende a centrarse en el surgimiento y desarrollo de nuevos conceptos ligados a una mayor transformación vinculada a la transparencia, la participación ciudadana y la colaboración, siendo el Gobierno Abierto el principal referente en este ámbito. Un concepto que plantea un cambio de paradigma en el modelo de relaciones entre el Gobierno y la sociedad, pero con todavía limitadas reflexiones sobre la necesidad de adaptar las organizaciones públicas y los profesionales que las integran.

Para contribuir a dicho debate el artículo propone una breve aproximación al concepto de Gobierno Abierto y, en clave de ámbito interno, una aproximación a las competencias digitales requeridas a los profesionales que integran las organizaciones públicas para afrontarlos.

Para ilustrar el desarrollo de estas competencias se propone la revisión de dos experiencias, una desarrollada por la Diputación de Barcelona, la Comunidad de Recursos Humanos (CORH) y otra impulsada por la Generalitat de Catalunya, Innogent, que pueden concebirse en términos de Gobierno Abierto y como modelo de gestión del conocimiento en red entre organizaciones públicas. Ambas experiencias resultan relevantes para ser consideradas como estudios de caso del sector público por combinar componentes de transparencia, participación y colaboración que se asocian a dicho paradigma, además de integrar el desarrollo de competencias digitales.

El análisis de ambas experiencias permite ilustrar la retroalimentación que se produce entre iniciativas innovadoras que integran componentes de Gobierno Abierto y las competencias requeridas -y que sus dinámicas de funcionamiento tienden a fomentar- para capacitar a las organizaciones públicas. Aunque los dos estudios de caso no permiten obtener resultados concluyentes, y menos en clave de causalidad, se infiere que el desarrollo de ambas iniciativas permite activar dinámicas que favorecen el despliegue de determinadas competencias digitales, que se revelan claves para el desarrollo del Gobierno Abierto.

\section{Gobierno abierto y cambios internos en la administración}

Los procesos de incorporación intensiva de las TIC al gobierno y a la Administración pública se asociaron, inicialmente, al concepto de Gobierno Electrónico (Criado y Ramilo, 2001; Salvador, 2007; Ramírez-Alujas, 2010). Según una de las definiciones más extendidas del concepto (Baum y Di Maio, 2000), el Gobierno Electrónico (equiparado posteriormente a Gobierno Digital) consiste en «the continuos optimisation of Government service delivery, citizen participation and governance by transforming internal and external realtionships though technology, the Internet and new media». 
Una definición que ponía el énfasis en la importancia de cambio y de las dimensiones donde éste debería producirse. Sin embargo el desarrollo de Gobierno Electrónico registró avances desiguales y, como apunta Heeks, «many e-Goverment projects fail to deliver their promises in terms of specifics outcomes» (citado por Luna \& Gil, 2011:329).

Con independencia de dichos resultados, desde el ámbito político pero también desde el ámbito académico, ha surgido con fuerza el concepto de Gobierno Abierto. Aunque, no existe un consenso en cuanto a su definición, sí hay cierta convergencia en cuanto a las tres dimensiones en las que se articula (Ramírez-Alujas, 2011; Gascó, 2014; Cruz-Rubio, 2015):

- Transparencia. Asociada a explicar de la acción de gobierno incluyendo la apertura de datos compatibles y reutilizables, que permitan conocer y controlar el funcionamiento de las instituciones públicas. Esta dimensión se vincula a la rendición de cuentas o accountability (Gascó, 2014; Cruz-Rubio, 2014).

- Participación. Asociada a fomentar la implicación de la ciudadanía, empresas, asociaciones y profesionales en la definición y evaluación de políticas y servicios públicos. Como apunta Calderón (2014) internet transforma la participación, permitiendo a un ciudadano contactar de forma directa con sus gobernantes y a éstos consultar permanentemente a la ciudadanía sobre las políticas. El uso de internet y de las redes sociales propicia el surgimiento de nuevas fórmulas de participación, pero exige que las administraciones públicas deban redefinir su funcionamiento y replantear la forma de hacer las cosas para poder responder a las expectativas de la sociedad.

- Colaboración. Asociada al desarrollo de fórmulas de trabajo conjunto y transversal entre diferentes actores dentro y fuera de la Administración, incluyendo actuaciones que comprenden iniciativas de interoperabilidad, innovación social y de coproducción (Gascó, 2014). Al relacionarse con la coproducción de servicios, la colaboración requiere la asunción de responsabilidad activa y constante por parte de los diferentes actores que participan.

A efectos del presente artículo se propone definir el concepto de Gobierno Abierto como un modelo de gobierno que, promoviendo la transparencia y la rendición de cuentas, la participación y la colaboración, y a través de un uso intensivo de las TIC, persigue obtener el máximo valor público potenciando la innovación (Calderon, 2014; Cruz-Rubio, 2014).

Para el desarrollo efectivo de dicho concepto se requieren cambios internos en las Administraciones Públicas que permitan capacitarlas para afrontar los retos que ello supone. El alcance y la profundidad de las transformaciones internas son proporcionales a la acepción del mismo que se adopte y, consecuentemente, al cambio institucional que se plantee alcanzar. En todo caso resulta ineludible que entre dichas transformaciones se incluya la potenciación de los profesionales que integran la Administración pública y, en especial, su capacitación en el ámbito de las denominadas competencias digitales. 


\section{Competencias digitales y gobierno abierto}

Aunque las TIC están siendo ampliamente utilizadas por las organizaciones públicas, el desarrollo de un nuevo paradigma de relaciones entre el gobierno y la ciudadanía va mucho más allá del simple uso de herramientas, puesto que implica repensar las organizaciones, los procesos y las competencias de los empleados públicos. La Agenda Digital para Europa 2020 (https://ec.europa.eu/digital-agenda/en/ict-enabled-public-sector-innovation) sitúa el Gobierno Abierto en una posición estratégica y plantea acciones concretas como la creación de plataformas interoperables, el mejor uso de estándares abiertos, la implementación o el despliegue de servicios digitales en áreas claves de interés público. El impulso del paradigma del Gobierno Abierto se plantea a través de iniciativas como la apertura de datos para facilitar la colaboración, esto es, el diseño y la producción y la prestación de servicios públicos. Las TIC deberían facilitar este tipo de iniciativas colaborativas y participativas así como el aumento de la transparencia, la corresponsabilidad y la rendición de cuentas.

Es en este nuevo marco de actuación donde el uso de las TIC representa un motor de transformación, unido al cambio en la cultura de las organizaciones públicas (Ballart y Ramió, 2000). Para ello es imprescindible no solo promover la incorporación de estas tecnologías sino también nuevos estilos en la gestión y, en especial, promover nuevas competencias de los profesionales al servicio de las organizaciones públicas.

El paradigma del Gobierno Abierto ofrece nuevas formas de participación y colaboración a la ciudadanía, en especial a partir de las denominadas tecnologías sociales que favorecen la implicación de una ciudadanía activa. Ello puede potenciar procesos de innovación pública no solo dentro de las organizaciones públicas sino también fuera de éstas, en especial gracias a la desintermediación de las actividades entre organizaciones e individuos. La cuestión es si los servidores públicos están preparados para afrontar los nuevos retos que plantean las tecnologías y las nuevas formas de relación (Criado \& Rojas, 2013).

El proceso de adaptación requiere planificación, estrategia y dirección (Gómez; 2013), pero también, y especialmente, capacitar a los recursos humanos en competencias relacionadas con las tecnologías digitales.

En una primera aproximación se puede definir el concepto de competencia digital como la «capacidad para la gestión del conocimiento tácito y explicito empoderada por la utilización de las TIC y el uso estratégico de la información. La e-competencia va más allá de la utilización de una TIC en particular, ya que también incluye conocimientos y actitudes orientadas al trabajo colaborativo, la innovación y el aprendizaje constante, así como la creación de nuevas ideas para enfrentar problemas desconocidos en diversos contextos» (Cobo, 2010: 4).

Según el Instituto de Tecnologías Educativas Departamento de Proyectos Europeos, la competencia digital consiste en «disponer de habilidades para buscar, obtener, procesar y comunicar información y para transformarla en conocimiento» (2011: 2). Ello implica integrar diferentes habilidades como la de acceder e interpretar la información, y facilitar su análisis y transmisión, incluyendo las TIC como elemento esencial para ello.

Estas competencias se asocian a rasgos personales como la autonomía, la eficacia, la responsabilidad, la actitud crítica y reflexiva para «seleccionar, tratar y utili- 
zar la información disponible, contrastándola cuando sea necesario y respetar las normas de conducta acordadas socialmente para regular el uso de la información y sus fuentes en los distintos soportes» (Instituto de Tecnologías Educativas Departamento de Proyectos Europeos, 2011: 2). En todo caso, más allá del desarrollo de habilidades ofimáticas, el desarrollo de competencias digitales integra el dominio de las TIC con la gestión de la información.

A partir de la identificación de competencias digitales por parte de diferentes autores (Salvatella \& Magro, 2014; Ferrari, 2013; Cobo, 2010; Fages-Ramió, 2008), para el sector público se pueden delimitar aquellas que se asocian al concepto de Gobierno Abierto, más allá del dominio de las herramientas TIC, y que se pueden concretar en:

1. Visión estratégica: asociada a comprender el fenómeno digital y la incorporación del mismo en la orientación de los proyectos de la organización.

2. Liderazgo en red: asociada a dirigir y coordinar equipos de trabajo distribuidos en red y entornos digitales, con una clara visión estratégica.

3. Gestión de la información: asociada a buscar, obtener, evaluar, organizar y compartir información en entornos digitales. Hace referencia tanto a la navegación, búsqueda, filtrado y evaluación de información como a su almacenamiento y recuperación.

4. Innovación y creatividad para afrontar los nuevos problemas: asociada a aportar ideas innovadoras, con una actitud proactiva, partiendo de la realidad digital de la organización.

5. Comunicación digital: asociada a comunicar, relacionar y colaborar de forma eficiente con herramientas en entornos digitales. Dentro de esta área de competencia digital se podrían desagregar diferentes subcompetencias: interacción a través de la tecnología, compartición de información y contenidos, implicación en procesos online para la ciudadanía, colaboración a través de canales digitales y gestión de la identidad digital, entre otras.

6. Trabajo en red: asociada a trabajar, colaborar y cooperar en entornos digitales, generando redes estables con agentes internos y externos a la organización. Se incluye la capacidad de trabajar de forma colaborativa, compartiendo recursos para generar valor y también la capacidad de utilizar las herramientas web 2.0 para crear redes y obtener beneficios profesionales colectivos a partir del conocimiento compartido.

7. Aprendizaje continuo: asociada a gestionar el propio aprendizaje de manera autónoma, conocer y utilizar recursos digitales, mantener y participar de comunidades de aprendizaje y estar constantemente actualizado.

8. Creación de contenidos: asociada a crear y presentar nuevos contenidos, incluyendo tanto el desarrollo de contenidos como la integración y reelaboración de información.

9. Resolución de problemas: asociada a la identificación de necesidades y recursos digitales para tomar decisiones informadas, utilizando de forma creativa las tecnologías, con una orientación a resolver problemas. Con ello 
se incluirían la resolución de problemas técnicos, la identificación de necesidades y respuestas basadas en la tecnología, la innovación y creatividad usando la tecnología y la identificación de brechas en competencias digitales.

10. Seguridad: asociada a velar por la protección de datos y de la identidad digital. En esta área se incluiría tanto la protección de dispositivos como la protección de datos personales.

A modo de conclusión, con carácter transversal, la mayor parte de competencias digitales se asocian a la denominada actitud 2.0 (Fages, 2008), entendida en términos de predisposición al uso de las herramientas para generar redes y crear valor en colectivo a partir de la gestión e intercambio de información y conocimiento.

El desarrollo de estas competencias se plantea para el conjunto de profesionales de la organización, pero obviamente adecuando su profundización a los contenidos, funciones y responsabilidades del puesto de trabajo ocupado.

En cuanto a las dinámicas vinculadas a la adquisición de estas competencias en las organizaciones, se plantea como esencial la combinación de acciones formativas con la generación de actividades que faciliten tanto su configuración concreta como su desarrollo efectivo adaptado a la realidad organizativa. En este sentido resulta sugerente analizar experiencias que integran actuaciones que pueden facilitar el desarrollo de algunas de las competencias digitales presentadas.

A modo de ilustración, en los siguientes apartados se presentan y se analizan dos experiencias de innovación vinculadas a la gestión de recursos humanos que integran componentes propios del Gobierno Abierto y que plantean actividades que pueden asociarse a las competencias digitales para su desarrollo.

\section{Dos experiencias considerar}

Para desarrollar a nivel aplicado los conceptos introducidos en el marco analítico se propone analizar dos experiencias vinculadas al ámbito de los recursos humanos en las Administraciones públicas que combinan diversas características asociadas al Gobierno Abierto y que integran competencias digitales en dicho proceso. Las dos experiencias seleccionadas son la Comunidad de Recursos Humanos de la Diputación de Barcelona (CORH) e Innogent del Departamento de Gobernación y Relaciones Institucionales de la Generalitat de Catalunya.

Las técnicas que se han utilizado para recabar y analizar la información de ambos casos son esencialmente cualitativas, combinando análisis documental con el seguimiento de las dos experiencias mediante contactos directos con las personas responsables de cada iniciativa.

La CORH es un programa transversal orientado a facilitar la generación de conocimiento compartido entre responsables de recursos humanos de los Ayuntamientos de la provincia de Barcelona. El programa se articula en torno a tres conceptos básicos: la gestión del conocimiento, el trabajo en red y la gestión de los recursos humanos. Su objetivo es impulsar una red presencial y una red virtual para compartir conocimiento y dotar de herramientas de apoyo a la toma de decisiones de los responsables de recursos humanos de las corporaciones locales implicadas. 
Innogent es un proyecto de generación de conocimiento compartido e innovación entre profesionales del sector público creado dentro de una línea de actuación del Plan de Reforma de la Administración de la Generalitat de Catalunya para la transformación de la gestión de los recursos humanos. Esta iniciativa se presenta tomando como valores de referencia la profesionalidad, el reconocimiento y la responsabilidad, y se concreta en un plan corporativo de fomento de la innovación.

Cuadro 1. Elementos constitutivos de las experiencias analizadas

\begin{tabular}{|c|c|c|}
\hline $\begin{array}{l}\text { ELEMENTOS DEL } \\
\text { PROYECTO }\end{array}$ & CORH & INNOGENT \\
\hline CREACIÓN & $\begin{array}{l}\text { Concebido a finales de } \\
\text { 2003. Implementado en marzo } 2005\end{array}$ & $\begin{array}{l}\text { Se enmarca en el Plan de Reforma } \\
\text { de la Administración de la } \\
\text { Generalitat de Catalunya y de su } \\
\text { sector público acordado el } 3 \text { de } \\
\text { junio de } 2014 \text { (Eje 6.Innovación y } \\
\text { Talento). } \\
\text { Implementado en Julio } 2014\end{array}$ \\
\hline $\begin{array}{l}\text { NECESIDAD A LA } \\
\text { QUE RESPONDE }\end{array}$ & $\begin{array}{l}\text { Crear un espacio para los agentes } \\
\text { relacionados con la gestión de } \\
\text { recursos humanos para intercambiar } \\
\text { conocimientos y experiencias. }\end{array}$ & $\begin{array}{l}\text { Crear un espacio para fomentar la } \\
\text { innovación en el sector público }\end{array}$ \\
\hline DEFINICIÓN & $\begin{array}{l}\text { Plataforma de profesionales del } \\
\text { sector público para impulsar el } \\
\text { desarrollo y know how de la } \\
\text { gestión de recursos humanos en las } \\
\text { administraciones públicas a } \\
\text { través de: } \\
>\text { Intercambio experiencias sobre } \\
\text { procesos de trabajo } \\
\text { relacionados con la gestión de } \\
\text { personal. } \\
\text { Homogeneización y mejora } \\
\text { prácticas gestión personal. } \\
>\text { Investigación especializada } \\
\text { sociedad conocimiento. } \\
\text { Para responder a falta de alianzas } \\
\text { entre responsables recursos humanos } \\
\text { en el mundo local }\end{array}$ & $\begin{array}{l}\text { Grupo colaborativo, interdisciplinar } \\
\text { e interdepartamental, formado por } \\
\text { profesionales de la Administración } \\
\text { de la Generalitat de Catalunya para } \\
\text { promover, detectar y poner en valor } \\
\text { prácticas innovadoras en el } \\
\text { funcionamiento de la } \\
\text { administración. }\end{array}$ \\
\hline
\end{tabular}

Fuente: Elaboración propia. 


\section{Cuadro 2. Instrumentos y actores de las experiencias analizadas}

\begin{tabular}{|c|c|c|}
\hline $\begin{array}{c}\text { ELEMENTOS } \\
\text { DEL } \\
\text { PROYECTO }\end{array}$ & CORH & INNOGENT \\
\hline $\begin{array}{l}\text { INSTRU- } \\
\text { MENTOS }\end{array}$ & $\begin{array}{l}\text { Un espacio virtual y un espacio } \\
\text { presencial. } \\
\text { - Espacio virtual con los siguientes } \\
\text { apartados: } \\
\text { - CORH, qué quién, dónde, cómo, } \\
\text { cuantos. } \\
\text { - Recopilatorio de bibliografía } \\
\text { - Legal, normativa en materia de } \\
\text { gestión de personal. } \\
\text { - Comunicaciones: foro, blog, } \\
\text { twiter, linkedin y listas de } \\
\text { distribución. } \\
\text { - Herramientas de trabajo (guía de } \\
\text { procedimientos, de puestos de } \\
\text { trabajo, indicadores de gestión, } \\
\text { etc.). } \\
\text { - Comparando, datos comparativos } \\
\text { de absentismo, dotación de } \\
\text { efectivos, etc. } \\
\text { - Recursos, (buenas prácticas, } \\
\text { convenios colectivos, etc.). } \\
\text { - Xarxa CORH. } \\
\text { - Otros. } \\
\text { Espacio presencial, compuesto por } \\
7 \text { grupos de trabajo, reuniones } \\
\text { trimestrales para compartir } \\
\text { información, conocimiento y } \\
\text { herramientas de trabajo. Se redacta } \\
\text { un acta de los temas tratados, de los } \\
\text { acuerdos se da acceso en el espacio } \\
\text { virtual. }\end{array}$ & $\begin{array}{l}\text { Estructuración en dos niveles: } \\
\text { - InnoBase: núcleo permanente que } \\
\text { define y diseña estrategias y } \\
\text { mecanismos para promover la } \\
\text { innovación (observatorio de } \\
\text { innovación, formación en técnicas } \\
\text { creativas, jornadas, acciones y actos de } \\
\text { reflexión sobre la creatividad e } \\
\text { innovación, colaboración con otras } \\
\text { entidades y grupos integrando } \\
\text { aportaciones de InnoXarxa y otros } \\
\text { agentes públicos o privados) } \\
\text { - InnoXarxa: grupo conector entre los } \\
\text { Departamentos de la Generalitat de } \\
\text { Catalunya e InnoBase para detectar } \\
\text { prácticas innovadoras. Los miembros } \\
\text { han de ser referentes departamentales } \\
\text { en innovación, sensibilizar sobre esta } \\
\text { cuestión y la mejora continua, valorar } \\
\text { propuestas, proponer indicadores de } \\
\text { impactos de políticas sobre la } \\
\text { ciudadanía, motivar prácticas } \\
\text { innovadoras, etc. } \\
\text { Tres ejes interrelacionados: } \\
\text { - Gestión del Talento.-fomentar el } \\
\text { intercambio de ideas. } \\
\text { - Gestión del conocimiento.-aumentar } \\
\text { el compromiso de las personas y su } \\
\text { motivación, fomentado nuevos } \\
\text { estilos de liderazgo y trabajo } \\
\text { colaborativo a través de la web, } \\
\text { cursos, compartiendo aprendizajes, } \\
\text { reflexionando sobre nuevas prácticas } \\
\text { y experiencias, participación, redes } \\
\text { expertos. etc. } \\
\text { - Gestión de la innovación.- fomentar } \\
\text { directamente aportaciones } \\
\text { innovadoras sin el filtro de origen } \\
\text { valorando todas las ideas por igual a } \\
\text { través de un canal (Innoteca). }\end{array}$ \\
\hline
\end{tabular}

Fuente: Elaboración propia.

\section{Análisis de las experiencias}

A partir de los referentes conceptuales considerados tanto en relación al Gobierno Abierto como a las competencias digitales, el análisis de las dos experiencias permite identificar una serie de rasgos destacados para caracterizar el desarrollo de capacidades organizativas. 
El primero de los casos, la CORH, institucionalizó una relación informal que existía previamente entre los directivos de recursos humanos de los Ayuntamientos de la provincia de Barcelona, pero aportando un nuevo impulso, un nuevo concepto y nuevos instrumentos. Entre los cambios generados cabe destacar la creación de una red presencial y virtual para la gestión del conocimiento en el ámbito de los recursos humanos de la Administración Pública.

Para ello se utilizaron las TIC, pero incorporando un enfoque de transparencia, participación de los miembros y lógica de colaboración orientada a generar aprendizaje y recursos compartidos. Estos rasgos permiten asociar la experiencia a componentes propios del Gobierno Abierto, aunque circunscrito inicialmente a las tres categorías de actores a los que se daba acceso a los distintos espacios.

Siguiendo dicho paradigma, la CORH planteó una nueva visión de la relación entre los profesionales vinculados a la gestión de los recursos humanos que dejaban de actuar de forma aislada e individual dentro de su organización para pasar a compartir conocimiento y generar recursos reutilizables con una lógica interorganizativa. Para ello se crearon grupos de trabajo y dinámicas de aprendizaje basados en la colaboración, con mayores grados de apertura que facilitaban nuevos escenarios de innovación.

Las dinámicas propiciadas por la CORH contribuyeron al desarrollo de buena parte de las competencias digitales identificadas en el apartado anterior. El análisis de los contenidos, la organización y los sistemas de intercambio que planteaba la plataforma virtual de la CORH permiten identificar cómo ésta se orientaba a potenciar la gestión de la información, la capacitación (mediante recopilatorios de bibliografía y legislación adecuados como recursos formativos y de aprendizaje continuo), o el trabajo colaborativo en red (con sistemas de comunicaciones como foros, blog y twitter). Los contenidos compartidos pueden interpretarse en términos de orientación a la resolución de problemas, a partir de la creación de nuevos contenidos y con una clara vocación innovadora para afrontar problemas mediante el aprendizaje continuo. Aunque los líderes de proyectos (seleccionados entre los miembros, categoría con mayor vinculación a la CORH) se responsabilizaban del desarrollo de propuestas en determinados ámbitos de contenidos, las competencias de visión estratégica y liderazgo en red no están especialmente desarrolladas en esta experiencia. La competencia vinculada a seguridad tan solo se consideraba al diferenciar diferentes tipos de actores implicados en la red, pero sin desarrollarla como tal entre los diferentes perfiles.

El énfasis de esta experiencia se sitúa en las competencias digitales vinculadas al trabajo colaborativo, la gestión del conocimiento y la comunicación digital.

La segunda experiencia considerada, Innogent, parte de un planteamiento que propone incorporar al empleado público en el proceso de transformación de la Administración, fomentando la innovación y el talento, propiciando el intercambio de ideas y de buenas prácticas, sin tener en cuenta la posición orgánica o la jerarquía que ocupan las personas que participan.

Para ello la iniciativa incorpora un componente tecnológico pero, más allá del mismo, plantea nuevos escenarios de actuación para dar respuesta a aspectos como la transparencia, la apertura de datos, el seguimiento, la evaluación y la redefinición de actuaciones. Se facilita el acceso a todos los empleados públicos a un canal virtual como herramienta de fomento de la cultura innovadora y de aprendizaje mutuo de forma directa (eludiendo jerarquías para priorizar las ideas). Aunque el proyecto se circunscribe a los empleados del sector público de la Generalitat de Catalunya, 
también se da cabida a la participación de agentes externos, públicos y privados, en una segunda instancia. Por el impacto de estos rasgos en términos de transparencia, participación y colaboración, se puede interpretar Innogent en términos de Gobierno Abierto.

Como planteamiento de red, Innogent pretende generar nuevos modelos de aprendizaje y transformar la cultura organizativa, implicando de forma activa a los empleados públicos en los procesos de transformación de la Administración autonómica, incidiendo también en su motivación.

En cuanto a las competencias digitales, el modelo de participación y colaboración que plantea Innogent se asocia al desarrollo de las vinculadas a la creación de contenidos y a la gestión de información, que se complementan con actividades formativas orientadas a fomentar la competencia de aprendizaje continuo. Para su funcionamiento operativo, Innogent presenta una plataforma virtual que se asocia a la gestión del conocimiento (fomentado el trabajo colaborativo a través de la web), la gestión del talento (mediante el fomento del intercambio de ideas) y la gestión de la innovación (mediante el espacio denominado Innoteca). Una articulación que favorece el desarrollo de las competencias de comunicación digital y trabajo en red. Las reglas del juego y la orientación proactiva hacia la mejora organizativa que sustentan la experiencia se plantean como referentes para el desarrollo de competencias como la innovación, la creatividad y la orientación a la resolución de problemas. Sin embargo no está tan claro que se desarrollen las competencias de visión estratégica y liderazgo en red, ni la de seguridad, al igual que en la experiencia anterior.

\section{Conclusiones}

La relectura de los dos estudios de caso a partir del marco analítico permite destacar diferentes elementos transversales que se proponen como aportación a la cuestión planteada inicialmente: las experiencias de innovación con un componente tecnológico que incorporen elementos del Gobierno Abierto requieren del desarrollo de competencias digitales, un elemento crítico para capacitar a las organizaciones públicas para que puedan afrontar los retos que plantea el nuevo paradigma.

Un primer aspecto a considerar es cómo cada una de las experiencias, además del componente tecnológico, incorpora una determinada acepción de Gobierno Abierto o, cuanto menos, algún componente asimilable al mismo. En ambas experiencias se avanza en transparencia y también en participación y colaboración, pero restringida a entornos profesionales vinculados a las organizaciones públicas. Aún así, en ambos casos se ofrecen espacios de transparencia, participación y colaboración a colectivos de diferentes ámbitos organizativos (o de diferentes organizaciones) sin mediar estructuras ni jerarquías. La combinación de dichos espacios con mecanismos y procesos de trabajo que facilitan la combinación de los tres componentes del Gobierno Abierto avalan la acepción del mismo en las dos experiencias consideradas.

Un segundo aspecto destacado es la apuesta que se realiza en ambos casos por el cambio en la cultura organizativa y en las reglas del juego, a través de la construcción de nuevas visiones respecto a la forma de trabajar y de relacionarse entre los actores implicados. En el caso de la CORH mediante institucionalización de las dinámicas de relación, aprendizaje e intercambio de recursos entre los gestores de 
recursos humanos. En el caso de Innogent, a través de la creación de un ecosistema de innovación, donde se valoran las ideas con independencia de la categoría profesional de las personas que las proponen, eludiendo el principio de jerarquía propio de la institución «función pública».

Un tercer aspecto a reseñar es que ambas experiencias plantean su estrategia superando las fronteras tradicionales de cada unidad u organización. En el caso de la CORH a través de la implicación de diferentes Ayuntamientos pero también, en las categorías de invitados, abriendo la interacción a otros actores públicos y privados. En el caso de Innogent, superando las habituales divisiones departamentales e incorporando también a otros actores públicos y privados.

En ambas experiencias la utilización de las TIC ha ido más allá de una mera incorporación de tecnología. En ambos casos se han replanteado las dinámicas de funcionamiento y se ha apostado por un cambio en la forma de trabajar. Por ello, un cuarto aspecto destacado, en la línea del argumento central del artículo, se refiere a cómo las dos experiencias proponen potenciar las competencias digitales, de forma alineada con la estrategia asociada a elementos propios del Gobierno Abierto. Tanto la CORH como Innogent han incorporado espacios virtuales donde se potencia la comunicación digital y el trabajo en red. La competencia de gestión de la información y la de aprendizaje continuo se propician en ambas experiencias a través de instrumentos concretos. En el caso de la CORH con herramientas como el recopilatorio de bibliografía y recursos para facilitar e-learning o espacios como InterCORH (una red social para facilitar la comunicación, compartir conocimiento, crear redes y mostrar experiencias y habilidades profesionales). En el caso de Innogent a través del eje de gestión del conocimiento, con cursos donde se favorece el aprendizaje compartido y la reflexión sobre nuevas prácticas. Un conjunto de recursos que a partir de la presentación y debate sobre iniciativas innovadoras plantean el desarrollo de competencias como la orientación a la innovación y a la creatividad o la orientación a la resolución de problemas.

Sin embargo, aunque ambas experiencias tienen como referente claro la innovación, no explicitan ni su apuesta por el Gobierno Abierto ni por el desarrollo de competencias digitales, lo que dificulta una articulación integrada de las mismas para reforzar capacidades institucionales en las Administraciones públicas implicadas.

A modo de balance final cabe destacar que ambas iniciativas, además de combinar elementos propios del Gobierno Abierto y asociar al mismo las competencias digitales, también plantean un cambio más profundo de las instituciones o reglas del juego en las correspondientes Administraciones públicas. Las dos iniciativas planten nuevos valores que se reflejan en cómo se conciben los roles otorgados a los empleados públicos y a su relación con la organización, promoviendo la innovación y la creatividad asociada a la solución de problemas, a la mejora organizativa, al conocimiento y generación de ideas más allá de las jerarquías o las posiciones orgánicas, entre otros.

Nuevos modelos que, en el marco de las estrategias de Gobierno Abierto, plantean transformaciones innovadoras para las cuales se requieren nuevos perfiles profesionales con nuevas capacidades y actitudes, que permitan afrontar los retos que se plantean a las Administraciones públicas. 


\section{Bibliografía}

Ballart, X y Ramió, C. (2000). Ciencia de la Administración. Editorial Tirant lo Blanc, Valencia.

Baum, C and Maio, A. D. (2000) Gartner's four phases of e-government model. Stanford. Gartner Group.

Calderón, C. (2014). «Participación: ¿Dónde está el ciudadano?», en Calderón, C. (coord.). Guía práctica para abrir gobiernos. Manual de «open government» para gobernantes y ciudadanos. Goberna América Latina. Escuela de Política y Alto Gobierno. Instituto Universitario de Investigación Ortega y Gasset, Madrid.

Cobo, C. (2010). «Cultura digital y nuevos perfiles profesionales: Desafíos regionales», en @TIC Revista d'Innovació Educativa, nº 5, pp 1-7.

Criado, J. I. y Ramilo, $M^{a}$ C. (2001). «E- Administración: ¿Un reto o una nueva moda para las administraciones del S. XXI? Algunos problemas y perspectivas de futuro en torno a internet y las tecnologías de la información y la comunicación en las administraciones públicas», en Revista Vasca de Administración Pública, vol. 61, $\mathrm{n}^{\circ}$ I, pp, 11-43.

Criado, J. I. y Rojas Martín, F. (2013). «Aproximación general sobre la adopción de las redes sociales en las administraciones públicas» en Las redes sociales digitales en la gestión y las políticas públicas. Avances y desafios para un gobierno abierto. Colecció Obres Digitals. EAPC. Generalitat de Catalunya, Barcelona.

Cruz-Rubio, C. N. (2014). Hacia el gobierno abierto: Una caja de herramientas. Departamento para la Gestión Pública Efectiva. Organización de los Estados Americanos.

Cruz-Rubio, C. N. (2015), «¿Qué es (y es no es) gobierno abierto? Una discusión conceptual», en Economía. Revista en Cultura de la Legalidad, n 8 , marzoagosto, pp 37-53.

Fages Ramió, R. (2008). «Actitud 2.0: la política más allá de los blogs». Monográfico IV Congreso Internet, Derecho y Política (IDP). Software social y Web 2.0: Implicaciones jurídico- políticas», en Revista d'Internet, Dret $i$ Política, UOC, $\mathrm{n}^{\mathrm{o}}$ 7, pp 19-25.

Ferrari, A. (2013). «DGICOMP: A framework for developing and understanding Digital competence in Europe», en Joint Reserch Centre. Institut for Prospective Technological Studies. European Commision. Europan Union.

Gascó, M. (2014). «Qué es gobierno abierto (y qué no lo es)», en Calderón, C. (coord.). Guía práctica para abrir gobiernos. Manual de "open government» para gobernantes y ciudadanos. Goberna América Latina. Escuela de Política y Alto Gobierno. Instituto Universitario de Investigación Ortega y Gasset, Madrid.

Gómez, J. M. (2013). «Las redes sociales, una potente herramienta al servicio del open goverment», en Las redes sociales digitales en la gestión y las políticas públicas. Avances y desafios para un gobierno abierto. Colecció Obres Digitals. EAPC. Generalitat de Catalunya, Barcelona.

Instituto de Tecnologías Educativas Departamento de Proyectos Europeos (2011). Competencia digital, Ministerio de Educación. Gobierno de España. Accesible en http://recursostic.educacion.es/blogs/europa [Consultado, el 23/11/2015]. 
Luna, L. F. y Gil-García, J. R. (2011). «Using institutional theory and dynamic simulation to understand complex e-Government phenomena», en Government Information Quarterly, $\mathrm{n}^{\mathrm{o}} 28$, pp 320-345.

Ramírez-Alujas, A. (2010). «Innovación en la gestión pública y open government (gobierno abierto). Una vieja nueva idea», en Revista Buen Gobierno, $\mathrm{n}^{\circ}$ 9, julio-diciembre, pp 2-35.

Ramírez-Alujas, A. (2011). «Gobierno abierto y modernización en la gestión pública. Tendencias actuales y el (inevitable) camino que viene. Reflexiones finales», en Revista Enfoques: Ciencia Política y Administración Pública, vol. IX, nº.15, diciembre, pp. 99-115.

Ramírez-Alujas, Alvaro. (2012). «Innovación en las organizaciones y servicios públicos: ¿El eslabón perdido?. Bases para la transición hacia un modelo de innovación abierta y colaborativa», en Estado, Gobierno, Gestión Pública. Revista Chilena de Administración Pública, $\mathrm{n}^{\circ}$ 19, pp. 5/50.

Salvador, M. (2007). «La dimensión interna del gobierno electrónico y el impacto de la e-Administración en la gestión de recursos humanos: ¿Están cambiando las reglas del juego?», en VIII Congreso de la Asociación Española de Ciencia Política y de la Administración (AECPA) Política para un Mundo en Cambio, 18-20 septiembre, Valencia.

Salvatella, J y Magro, C. (coord.). (2014). «Cultura digital y transformación de las organizaciones. Competencias digitales para el éxito profesional». Roca Salvatella, Barcelona. 
